Models for Image Analysis 



\title{
Recognizing Arithmetic Straight Lines and Planes
}

\author{
Jean Françon, Jean-Maurice Schramm, Mohamed Tajine \\ Laboratoire des Sciences de l'Image, d'Informatique et de Télédétection \\ Dépt. Informatique, 7, rue René Descartes, F-67084 Strasbourg cedex \\ email: $\{$ francon, jms, tajine $\} @ \mathrm{dpt}$-info.u-strasbg.fr
}

\begin{abstract}
The problem of recognizing a straight line in the discrete plane $\mathbb{Z}^{2}$ (resp. a plane in $\mathbb{Z}^{3}$ ) is to find an algorithm deciding wether a given set of points in $\mathbb{Z}^{2}$ (resp. $\mathbb{Z}^{3}$ ) belongs to a line (resp. a plane). In this paper the lines and planes are arithmetic, as defined by Reveilles [Rev91], and the problem is translated, for any width that is a linear function of the coefficients of the normal to the searched line or plane, into the problem of solving a set of linear inequalities. This new problem is solved by using the Fourier's elimination algorithm. If there is a solution, the family of solutions is given by the algorithm as a conjunction of linear inequalities. This method of recognition is well suited to computer imagery, because any traversal algorithm of the given set is possible, and also because any incomplete segment of line or plane can be recognized.
\end{abstract}

Key Words : Discrete plane, discrete line, recognition algorithm, Fourier's algorithm.

\section{Introduction}

The recognition of discrete straight lines or planes is a classical problem in computer imagery. It has been treated by numerous authors (see [Kro-Toc89, Kov90, Sto-Tos91, Deb-Rev94a, Deb-Rev94b, Deb95] and their references). In this paper, we consider that the adequate framework of this problem is the arithmetic geometry introduced by Reveilles [Rev91, Deb-Rev94c]. This theory leads to original and particularly interesting geometric solutions for the recognition problem of naive planes and of lines of several width [Deb-Rev94a, Deb-Rev94b, Deb95]. In this paper, we obtain algebraic solutions for a wider class of arithmetic lines and planes.

An arithmetic plane is the set of points $(x, y, z)$ of $\mathbb{Z}^{3}$ satisfying the inequalities $0 \leqslant a x+b y+c z+d<\omega$, where all parameters are integers and $\omega>0$. For $z=0$ it defines an arithmetic line in $\mathbb{Z}^{2}$. For a line, the rational $-a / b$ is called the slope. The parameter $\omega$ is called the (arithmetic) width of the plane or the line. The triplet $(a, b, c)$ for a plane, (resp. the pair $(a, b)$ for a line), is called the normal; the integers $a, b, c, d$ are called the coefficients of the plane or line. 
If $\omega=|a|+|b|+|c|$ for a plane (resp. $\omega=|a|+|b|$ for a line) then the plane (resp. line) is called standard. If $\omega=\max (|a|,|b|,|c|)$ for a plane (resp. $\omega=\max (|a|,|b|)$ for a line) then the plane (resp. line) is called naive.

Naive lines are classical 8-connected straight lines. Naive planes are their 3D extension, and are essentially the discrete planes of the litterature. Standard lines are essentially the 4-connected "digital straight lines" of [Kov90]. Standard planes are their 3D extension; they are studied in [Fra96]; note that the surfel boundary of a voxel object is locally a segment of a standard plane. The interest of the concept of width lies in the unification of several notions.

In this paper, the recognition problem is the following : assume we are given a set $P=\left\{p_{1}, p_{2}, \ldots, p_{n}\right\}$ of $n$ points of $\mathbb{Z}^{2}$ (resp. $\mathbb{Z}^{3}$ ), does it exist an arithmetic line (resp. plane) of width $\omega$, a given linear function of the coefficients, in which $P$ is included ? If the answer is positive, what are the normals of the solutions? This statement of the problem is more general than the classical one. For lines, the classical assumption is that we are given a sequence of 8-connected or 4-connected points, and not a set. The non usual linear condition on the width will be explained later.

The solution we propose consists in translating the problem into a system of linear inequalities whose unknowns are the coefficients; in fact, a system of $2 n+2$ (resp. $2 n$ +3 ) inequalities with 3 (resp. 4) unknowns $a, b, d$ (resp. $a, b, c, d$ ). The geometric problem is equivalent to the existence of a solution of this system. If a solution exists, then the normals of the solutions are obtained by eliminating the unknown $d$, thus obtaining a set of linear inequalities to be satisfied by the coefficients. The existence problem is solved by an elimination algorithm, due to Joseph Fourier, also known as Fourier-Motzkin algorithm, analogous to the classical Gauss elimination algorithm for linear equalities. If this process ends with a contradiction, then the system has no solution; if it ends without contradiction in $\mathbb{R}$, then the system of inequalities has a solution in $\mathbb{Z}$.

The use of linear inequations for the recognition problem is also that of [StoTos91] for a restricted problem. In this paper, other methods of resolution are used. Fourier algorithm was also used by [Ver94] for a connected problem on discrete planes. The possibility of a linear algorithm was pointed out in these papers. Here we do not study the complexity, leaving it for another paper together with some developments.

In section 2 , we translate the geometric problem into the algebric problem. In section 3 we describe the Fourier algorithm and related algorithmic questions. The results of several examples are given in section 4 , before a short conclusion.

\section{The linear inequations system}

First, we explain the method for lines; then we give the result for planes. Without loss of generality, we search for an arithmetic line $D$ of width $\omega$ defined by the inequalities:

$$
0 \leqslant a x+b y+d<\omega, \quad 0 \leqslant a \leqslant b .
$$

Thus, we are searching for $D$ in one octant of the $(a, b)$ space. 
Let $P=\left\{\left(x_{1}, y_{1}\right),\left(x_{2}, y_{2}\right), \ldots,\left(x_{n}, y_{n}\right)\right\}$ be a set of $n$ points in $\mathbb{Z}^{2}$. Then $P$ is included in $D$ iff all the inequations $0 \leqslant a x_{i}+b y_{i}+d<\omega$ hold. Thus, the searched line $D$ exists iff the following system of $2 n+2$ inequalities of unknowns $a, b, d$ has a solution :

$$
0 \leqslant a \leqslant b, \quad 0 \leqslant a x_{i}+b y_{i}+d<\omega, \quad 1 \leqslant i \leqslant n .
$$

Assume now that $\omega$ is a linear function of $a$ and $b$. For example, $\omega=b$ if we are searching for a naive line, or $\omega=a+b$ for a standard line. That is, we fix a family of lines of given width. The system $\left(^{*}\right)$ can now be solved by known methods. It has a solution iff the system $\left({ }^{*}\right)$ obtained by eliminating $d$ has a solution :

$$
\text { (**) } 0 \leqslant a \leqslant b,-a x_{i}-b y_{i}<\omega-a x_{j}-b y_{j}, 1 \leqslant i, j \leqslant n .
$$

This system has $n^{2}+2$ inequalities and 2 unknowns. In numerous examples, it is very redundant. If a solution exists for $(*)$, then the set of normals of all the solutions is characterized by the inequations $(* *)$.

\section{Example}

The simplest example is the search for a naive line joining two given points : $(0,0)$ and $(x, y)$. Assume $x>0$. Then the system $\left(^{*}\right)$ is

$$
0 \leqslant a \leqslant b, \quad 0 \leqslant d<b, \quad 0 \leqslant a x+b y+d<b .
$$

It has a solution iff the system (**), obtained by elimination of $d$, has one :

$$
0 \leqslant a \leqslant b, \quad 0<b, \quad 0<b-a x-b y, \quad-a x-b y<b .
$$

This can be written in a more familiar form, by using the slope $-a / b$ of $D$ :

$$
0 \leqslant a \leqslant b, \quad 0<b,(y-1) / x<-a / b<(y+1) / x .
$$

This system defines the set of solutions in the $(a, b)$ space.

For the recognition of a plane, we search for a plane $D$ in the cone $0 \leqslant a \leqslant b \leqslant c$ and of width $\omega$, a given linear function of $a, b$, and $c$. We are given a set of $n$ points in $\mathbb{Z}^{3}$. We obtain a system of $2 n+3$ linear inequalities with unknowns $a, b, c, d$. If a solution exists, then by eliminating $d$ we get the set of solutions defined by $n^{2}+3$ linear inequalities linking $a, b$ and $c$ which caracterize the normals.

\section{Fourier's elimination algorithm}

\section{1. An outline of history of Fourier's method and its extensions}

Fourier's method was published 1826. It has been rediscovered a number of times by different authors: Motzkin [Mot36] (the name Fourier-Motzkin's elimination algorithm is often used for this method) Fan [Fan56], Dantzig [Dan63], Kuhn [Kuh56]. (Some authors also refered to this method as Kuhn-Fourier elimination algorithm) .

The Fourier's method is used for solving a system of linear constraints of the form $a_{1} x_{1}+\ldots+a_{n} x_{n}>b$ on the set of real numbers (or more generally on an ordered field) where $\succ$ is $>, \geqslant$ or $=$, and $a_{1}, \ldots, a_{n}, b$ are real numbers.

The Fourier's method is a special case of the Tarski's algorithm [Tar 51] which considers any sentence of first order logic with atomic formula of the form : $P\left(x_{1}, \ldots\right.$, $\left.x_{n}\right) \succ 0$ where $P\left(x_{1}, \ldots, x_{n}\right)$ is a polynomial over $\mathbb{R}$. 
An integer Fourier's elimination algorithm (for solving linear constraints in $\mathbb{Z}$ ) can be obtained as a special case of the Presburger's algorithm [Pre29] which considers any sentence of first order logic with linear constraints over $\mathbb{Z}$ as atomics formulas. If we replace linear constraints by polynomial constraints over $\mathbb{Z}$ as in the Tarski's algorithm, the problem is undecidable as it has been proved by Matiiassevitch [Mat70].

\subsection{The Algorithm :}

Below, we assume that the relation $>$ is $>$ or $\geqslant$.

Let $S_{\mathrm{n}}$ be a system of the form :

$$
\begin{aligned}
& a_{1,1} x_{1}+\ldots+a_{1, n} x_{n} \succ_{1} b_{1} \\
& a_{2,1} x_{1}+\ldots+a_{2, n} x_{n} \succ_{2} b_{2} \\
& \ldots \\
& a_{m, 1} x_{1}+\ldots+a_{m, n} x_{n} \succ_{m} b_{m}
\end{aligned}
$$

where $a_{i, j}, b_{i}$ are real numbers and $>_{i}$ is $>$ or $\geqslant$, for $1 \leqslant i \leqslant n$ and $1 \leqslant j \leqslant m$.

The Fourier's elimination algorithm consists in successive eliminations of the unknowns. Each step transforms the constraints system $S_{n}$ with the unknowns $x_{1}, \ldots, x_{n}$ to a new system $S_{n-1}$ in which one of the unknowns, say $x_{n}$, does not occur anymore : $x_{n}$ has been eliminated. $S_{n-1}$ is obtained from $S_{n}$ by using the following combination of the constraints :

- All the constraints of $S_{n}$ in wich $x_{n}$ does not occur $\left(a_{i, n}=0\right)$ are in $S_{n-1}$.

- For all $1 \leqslant i, j \leqslant \mathrm{~m}$ such that $a_{i, n}>0$ and $a_{j, n}<0$, the constraint

$$
\left(a_{i, n} a_{j, 1}-a_{j, n} a_{i, 1}\right) x_{1}+\ldots+\left(a_{i, n} a_{j, n-1}-a_{j, n} a_{i, n-1}\right) x_{n-1} \succ_{i, j} \quad a_{i, n} b_{j}-a_{j, n} b_{i},
$$

where $\succ_{i, j}$ is $\geqslant$ if $\succ_{i}$ and $\succ_{j}$ are $\geqslant$, and $>$ otherwise, is in $S_{n-1}$.

The system $S_{n}$ and $S_{n-1}$ are linked together by the fact that the set of solutions of $S_{n-1}$ is the projection over $\mathbb{R}^{n-1}$ of the set of solutions of $S_{n}$. It results that $S_{n}$ has a solution iff $S_{n-1}$ has a solution.

Finally, all the unknowns are eliminated : the system $S_{0}$ (eventualy empty) does not depend anymore on any unknown. It results from this that all the constraints in $S_{0}$ are inequalities over the real numbers and can only be true or false. It becomes trivial to prove the existence or inexistence of solutions for $S_{0}$, and, consequently for $S_{n}$.

\subsection{Some facts about Fourier's elimination algorithm}

- If for some $i, S_{i}$ contains a constraint of the form $(0 \geqslant d$ or $0>d)$ where $d$ is a strictly positive number or a constraint $(0>0)$, then the initial system $\left(S_{n}\right)$ is unsolvable; thus $S_{n}$ is unsolvable iff $S_{0}$ contains a constraint of the last type.

- If for some $i$, in all the constraints of $S_{i}$ the coefficients of $x_{i}$ have the same sign, or if $S_{i}=\phi$, then $S_{n}$ is solvable in $\mathbb{R}$.

- The set of solutions of $S_{n}$ is a polyhedral set of $\mathbb{R}^{n}$.

- If $S_{n}$ is a homogeneous system of linear constraints ( $b_{i}=0$ for $1 \leqslant i \leqslant m$ ), then the set of solutions of $S_{n}$ is a cone of $\mathbb{R}^{n}$. In this case, if $\left(c_{1}, \ldots, c_{n}\right)$ is a solution of $S_{n}$ and $\lambda$ is a strictly positif number then $\left(\lambda c_{1}, \ldots, \lambda c_{n}\right)$ is also a solution of $S_{n}$; so, if $S_{n}$ is solvable in $\mathbb{R}$ then $S_{n}$ has a solution in $\mathbb{Z}^{n}$. 
Example 1.

$$
\begin{array}{rr}
S_{3} & \multicolumn{1}{c}{S_{2}} \\
x_{1}+x_{2}+x_{3}>0 & x_{1}+x_{2}>0 \\
-x_{3} \geqslant 0 & x_{1}>0 \\
-x_{2}-x_{3} \geqslant 0 & x_{2}>0 \\
-x_{1}-x_{3}>0 &
\end{array}
$$

All coefficients of $x_{2}$ in $S_{2}$ are $\geqslant 0$, thus the initial system $\left(S_{3}\right)$ is solvable in $\mathbb{R}$.

Example 2.

$$
\begin{array}{rrr}
S_{3} & S_{2} & \multicolumn{1}{c}{S_{1}} \\
x_{1}+x_{3}>0 & x_{1}>0 & x_{1}>0 \\
x_{2}+x_{3} \geqslant 0 & -x_{2}>0 & -x_{1}>0 \\
-x_{3} \geqslant 0 & x_{2} \geqslant 0 & 0>0 \\
-x_{1}-x_{2}-x_{3}>0 & -x_{1}>0 &
\end{array}
$$

The system $S_{1}$ contains a contradiction $(0>0)$, thus the initial system $\left(S_{3}\right)$ is unsolvable in $\mathbb{R}$.

\section{Definitions}

Let $E$ be a subset of $\mathbb{R}$. Let $S$ be a set of linear constraints and $C$ be a linear constraint, we say that $C$ is a consequence of $S$ in $E$, which we denote by $S \mid=_{E} C$, if the set of solutions of $S$ in $E^{n}$ is a subset of the set of solutions of $C$ in $E^{n}$.

Let $S, S^{\prime}$ be two sets of linear constraints, we say that $S$ and $S^{\prime}$ are equivalent in $E$ which we denote by $S|=|_{E} S^{\prime}$, if $\forall C \in S^{\prime}, S \mid=_{E} C$ and $\forall C \in S, S^{\prime} \mid=_{E} C$ hold .

A set $S$ of linear constraints is said to be minimal over $E$ if for all $C$ in $S, C$ is not a consequence of $S-\{C\}$ in $E . S$ is said minimal if it is minimal over $\mathbb{R}$.

\section{Proposition 1}

- $\quad S \mid=_{\mathbb{R}} C$ iff the application of the Fourier's elimination algorithm to $S \cup\{\neg(C)\}$ leads to contradiction (i.e. $S \cup\{\neg(C)\}$ is unsolvable ), where

$$
\neg(C)=-a_{1} x_{1}-\ldots-a_{n} x_{n} \geqslant 0 \text { if } C=a_{1} x_{1}+\ldots+a_{n} x_{n} \geqslant 0 \text { else }-a_{1} x_{1}-\ldots-a_{n} x_{n}>0 .
$$

- If $E^{\prime} \subseteq E$, then $\left(S||_{E} C \Rightarrow S \mid=_{E^{\prime}} C\right)$, thus $\left(S||_{\mathbb{R}} C \Rightarrow S||_{\mathbb{Z}} C\right)$.

- If $\left.S\right|_{\mathbb{R}} S^{\prime}$ and $\left.S\right|_{\mathbb{R}} S^{\prime \prime}$ and $S^{\prime}, S^{\prime \prime}$ are minimals then $\operatorname{Card}(S)=\operatorname{Card}\left(S^{\prime \prime}\right)$, actually, $S^{\prime}$ defines the boundary of the set of solutions of $S$ in $\mathbb{R}^{n}$.

\subsection{Integer Fourier's elimination algorithm}

Fourier's elimination algorithm can be adapted for solving a system of linear inequalities in $\mathbb{Z}$. At each step the introduction of the "or" connector can be necessary and the size of the new system depends on the value of the coefficients.

Below, we present an algorithm for solving special systems of linear inequalities in $\mathbb{Z}$ which do not necessite the introduction of the "or" connector. This algorithm will be used for the study of the properties of the naive planes. 


\section{Proposition 2}

Consider the system $S_{n}$ :

$$
\begin{aligned}
& a_{1,1} x_{1}+\ldots+a_{1, n} x_{n} \succ_{1} 0 \\
& a_{2,1} x_{1}+\ldots+a_{2, n} x_{n} \succ_{2} 0 \\
& \ldots . . \\
& a_{m, 1} x_{1}+\ldots+a_{m, n} x_{n} \succ_{m} 0
\end{aligned}
$$

such that for $1 \leqslant i \leqslant n$ and $1 \leqslant j \leqslant m, a_{i, j}$ are integers, $>_{i}$ is $>$ or $\geqslant$, and $a_{i, n} \in\{-1,0,1\}$. Let $S_{n-1}^{\prime}$ be the system on the unknowns $x_{1}, \ldots, x_{n-1}$ obtained from $S_{n}$ by using the following combination of the constraints :

- all the constraints of $S_{n}$ in wich $x_{n}$ does not occur $\left(a_{i, n}=0\right)$ are in $S_{n-1}^{\prime}$;

- for all $1 \leqslant i, j \leqslant m$ such that $a_{i, n}=1$ and $a_{j, n}=-1$, the constraint $\left(a_{j, 1}+a_{i, 1}\right) x_{1}+\ldots+\left(a_{j, n-1}+a_{i, n-1}\right) x_{n-1} \succ_{i, j} \quad r_{i, j}$

where $\succ_{i, j}$ is $\geqslant$ if $\succ_{i}, \succ_{j}$ are both $\geqslant$, otherwise $\succ_{i, j}$ is $>$,

and $r_{i, j}=1$ if $\succ_{i}, \succ_{j}$ are both $>$, otherwise $r_{i, j}=0$

is in $S_{n-1}^{\prime}$.

Then, the set of solutions of $S_{n-1}^{\prime}$ in $\mathbb{Z}^{n-1}$ is the projection of the set of solutions of $S_{n}$ in $\mathbb{Z}^{n}$.

\section{Proof}

Let $\left(c_{1}, \ldots, c_{n}\right)$ be a solution of $S_{n}$ in $\mathbb{Z}^{n}$, then it is trivial that $\left(c_{1}, \ldots, c_{n-1}\right)$ is a solution of $S_{n-1}^{\prime}$ in $\mathbb{Z}^{n-1}\left(S_{n} \Rightarrow S_{n-1}^{\prime}\right)$.

Let $\left(c_{1}, \ldots, c_{n-1}\right)$ be an integer solution of $S_{n-1}^{\prime}$, then the last fact (in 3.3) implies that there exists $c_{n}$ in $\mathbb{R}$ such that $\left(c_{1}, \ldots, c_{n-1}, c_{n}\right)$ is a solution of $S_{n}$ in $\mathbb{R}^{n}\left(S_{n-1}^{\prime} \Rightarrow S_{n-1}\right)$. We will prove that we can choose for $c_{n}$ an integer value.

If $a_{i, n}=1$ and $a_{j, n}=-1$, then $a_{j, 1} c_{1}+\ldots+a_{j, n-1} c_{n-1} \succ_{j} c_{n} \succ_{i}-\left(a_{i, 1} c_{1}+\ldots+c_{i, n-1} b_{n-1}\right)$.

Let $l, l^{\prime}, a, b$ such that $\left.a=-\left(a_{l, 1} c_{1}+\ldots+a_{l, n-1} c_{n-1}\right)=\max \left\{-\left(a_{i, 1} c_{1}+\ldots+a_{i, n-1} c_{n-1}\right)\right) \mid a_{i, n}=1\right\}$, $b=a_{l, 1} c_{1}+\ldots+a_{l, n-1} c_{n-1}=\min \left\{a_{i, 1} c_{1}+\ldots+a_{i, n-1} c_{n-1} \mid a_{i, n}=-1\right\}$.

Then, we have $b \succ_{l} c_{n} \succ_{l^{\prime}} a$, so, if $\succ_{l}$ and $\succ_{l}$ are $>$ then $b-a>1$, thus $c_{n}=a+1$ is suitable, else $c_{n}=a$ or $c_{n}=b$ is suitable.

\section{Results}

The Fourier's algorithm has been programmed and it produced the following results (the simplest ones can be checked by hand).

\subsection{Lines}

\subsubsection{Example of recognition of naive lines}

Let $P_{1}=\{(0,0),(1,0),(4,-1),(6,-2),(8,-3)\}$. Then using the Fourier's elimination algorithm, we prove the existence of naive lines (with $\omega=b$ ) containing $P_{1}$, and caracterize it by the minimal conditions $c \geqslant 0,-a+b-c>0,-4 a+2 b-c>0,8 a-3 b+c \geqslant 0$. 


\subsubsection{Example of recognition of standard lines}

Let $P_{2}=\{(0,0),(1,0),(3,0),(4,-1),(5,-1),(6,-2),(8,-3)\}$. Then using the Fourier's elimination algorithm, we prove the existence of standard lines (with $\omega=a+b$ ) containing $P_{2}$, and caracterize it by the minimal conditions :

$$
c \geqslant 0,-2 a+b-c>0,8 a-3 b+c \geqslant 0 \text {. }
$$

\subsubsection{Example of recognition of thick lines}

Let $P_{3}=\{(0,0),(1,0),(3,0),(4,-1),(5,-1),(6,-2),(8,-3),(0,-1),(8,-4)\}$. Then using the Fourier's elimination algorithm, we prove the existence of thick lines (with $\omega=a+2 b, 0 \leqslant a \leqslant b$ ) containing $\mathrm{P}_{3}$, and caracterize it by the minimal conditions :

$$
-2 a+2 b-c>0,-b+c \geqslant 0,8 a-4 b+c \geqslant 0 .
$$

\subsection{Planes}

We always assume that the coefficients of the searched plane satisfy the inequalities $0 \leqslant a \leqslant b \leqslant c$.

\subsubsection{Naive planes}

Let us recall that a naive plane is functional, that is, for any $(x, y)$ in $\mathbb{Z}^{2}$ there exists one and only one $z$ such that $(x, y, z)$ belongs to the plane.

Let us call bicube a set of 4 points of a naive plane of the form

$$
\left\{\left(x, y, z_{1}\right),\left(x+1, y, z_{2}\right),\left(x+1, y+1, z_{3}\right),\left(x, y+1, z_{4}\right)\right\} \text {. }
$$

Proposition 3. In the set of naive planes of normal $(a, b, c)$ there exist only 5 distinct bicubes (up to a translation), and at most 4 in a given plane; i.e. :
(i) $\{(x, y, z),(x+1, y, z),(x+1, y+1, z),(x, y+1, z)\}$
iff $a+b<c$,
(ii) $\{(x, y, z),(x+1, y, z),(x+1, y+1, z-1),(x, y+1, z)\}$
iff $0<a$ and $a+b<2 c$,
(iii) $\{(x, y, z),(x+1, y, z),(x+1, y+1, z-1),(x, y+1, z-1)\} \quad$ iff $a<b$,
(iv) $\{(x, y, z),(x+1, y, z-1),(x+1, y+1, z-1),(x, y+1, z-1)\}$ iff $0<a$ and $b<c$,
(v) $\{(x, y, z),(x+1, y, z-1),(x+1, y+1, z-2),(x, y+1, z-1)\}$ iff $a+b>c$.

A tricube [Deb95] is a set of 9 points of a naive plane whose projection onto the $(\mathrm{x}, \mathrm{y})$ plane is a point together with its 8-neighbours. It has been shown [Deb95] that, in the set of naive planes, there exist only 40 distinct tricubes (up to a translation), and [Rev95] at most 9 in a given plane. These concepts have some importance because of the following result (see [Fra95, Fra96] for definitions and an analogous theorem for standard planes).

\section{Proposition 4.}

A naive plane has the structure of a 2-dimensional combinatorial manifold without boundary, whose faces are bicubes and whose umbrella are tricubes.

The table in the Appendix gives, for each of the forty tricubes, the necessary and sufficient conditions on the normals of the planes containing these tricubes, computed by Fourier's elimination algorithm. The $3 \times 3$ table of integers of this Appendix are to be read as follows : the value $z$ in column $x$ ( $x$ is increasing by one from left to right) and line $y$ ( $y$ is increasing by one from bottom to top) is such that $(x, y, z)$ belongs to a tricube. 


\subsubsection{Example of recognition of standard planes}

Let $P=\{(0,0,0),(0,1,0),(0,1,1),(0,0,1),(1,0,0),(1,1,0),(1,0,1),(2,0$, $0),(2,1,0),(2,0,1),(3,0,0),(3,1,0),(3,0,1),(4,0,0),(4,1,0),(4,0,1),(5,0,0)$, $(5,1,0),(5,0,1)\}$. Then by using the Fourier's elimination algorithm, we prove the existence of standard planes (with $\omega=a+b+c$ ) containing $P$, and caracterize it by the minimal conditions : $d \geqslant 0, a-d>0,-4 a+b-d>0,-4 a+c-d>0$.

\section{Conclusion}

We have shown how to recognize an arithmetic straight line or plane, given a set of its points. This method works under the only restriction of a width depending linearly on the coefficients of the normal. Thus, it works for partially known segments of lines or planes, of any width (naive, standard or other). Furthermore, the data being a set of points, any order of traversal of these points can be used in an incremental recognition algorithm.

It is now possible to answer numerous theoretical, algorithmic, and practical open questions. First, how to facetize the boundary of a voxel object (see [Bor-Fr94] for the precise statement). This problem will be solved soon.

\section{Acknoledgements}

This work has gained by fruitful discussions with J.P. Reveilles, I. Debled, Ph. Borianne, J.M. Chassery, and of numerous members of the LSIIT.

\section{References}

[Bor-Fra94] Ph. Borianne et J. Françon. Polyhédrisation réversible de volumes discrets. $4^{\text {th }}$ Conference on Discrete Geometry in Computer Imagery, Grenoble, 1994.

[Dan63] G. B. Dantzig. Linear programming and extentions. Princeton, 1963.

[Deb95] I. Debled-Renesson. Etude et reconnaissance des droites et plans discrets. Thèse de doctorat soutenue à l'Université Louis Pasteur de Strasbourg , 1995.

[Deb-Rev94a] J.P. Reveilles, I. Debled-Renesson. A linear algorithm for segmentation of discrete curves. Third International Workshop on Parallel Image Analysis: Theory and Applications, Washington, 1994.

[Deb-Rev94b] I. Debled-Renesson, J.P. Reveilles. An incremental algorithm for digital plan recognition. $4^{\text {th }}$ Conference on Discrete Geometry in Computer Imagery, Strasbourg, 1994.

[Deb-Rev94c] I. Debled, J.P. Reveillès. A new approach to digital planes. Vision Geometry III, Boston, 1994.

[Fan56] K. Fan. On system of linear inequalities. In Kuhn-Tucker (eds.), Linear inequalities and related systems, Ann. of Math. Study 38: Princeton Univ. Press, 1956, p. 99-156.

[Fou 1826] J. B. J. Fourier. Solution d'une question particulière du calcul des inégalités. Oeuvre II, Paris, 1826, p. 317-328.

[Fra95] J. Françon. Arithmetic planes and combinatorial manifolds. $5^{\text {th }}$ Conference on Discrete Geometry in Computer Imagery, Clermont-Ferrand, 1995.

[Fra96] J. Françon. Sur la topologie d'un plan arithmétique. Theor. Comput. Sc. 156, 1996, p. 159-176. 
[Kov90] V.A.Kovalesky. New definition and fast recognition of digital straight line segments and arcs. Proc. of the $10^{\text {th }}$ Intern.Conf.on Pattern Recognition, Atlantic City, IEEE Press, vol. II, 1990, p.31-34.

[Kro-Toc89] W.G. Kropatsch, H. Tockner. Detecting the straightness of digital curves in $\mathrm{O}(\mathrm{N})$ steps. Computer Vision, Graphics, and Image Processing 45, 1989, p.1-21.

[Kuh56] H. W. Kuhn. Solvability and consistency for linear equations and inequalities. Amer. Math. Monthly, Vol 63, 1956, p.217-232.

[Mat70] Yu. Matiiassevitch. Enumerable sets are diophantine. Doklady Akad. Nauk SSSR, 191, 1970, p.279-282. (English translation : Soviet Math. Doklady, 1970, p. 354-357.

[Mot36] T. S. Motzkin. Beitrage zur theorie der linearen ungleichungen. Azriel, Jerusalem, 1936.

[Pre29] M. Presburger. Üeber die vollständigkeit eines gewissen systems der arithmetik ganzen Zahlen in welchen die addition als einziger aperation hervortritt. Comptes Rendus du $I^{\text {er }}$ Congrès des Math. des Pays Slaves, Warsaw, 1929, p. 92-101. [Rev91] J.P. Reveillès. Géométrie discrète, calcul en nombres entiers et algorithmique. Thèse d'état soutenue à l'Université Louis Pasteur, 1991.

[Rev95] J.P. Reveilles. Combinatorial pieces in digital lines and planes. Vision Geometry 4, SPIE'95, San Diego, 1995.

[Sto-Tos91] I. Stojmenovic, R. Tosic. Digitization schemes and the recognition of digital straight lines, hyperplanes, and flats in arbitrary dimensions. Contemporary Math., Vol. 119,1991, p. 197-212.

[Tar51] A. Tarski. A decision method for elementary algebra and geometry. Tech. Rep., University of California Press, Berkeley and Los Angeles, 1951.

[Ver94] P. Veelaert. Digital planarity of rectangular planar segments. IEEE Trans. on P.A.M.l., 16, 1994, p. 647-652. 


\section{Appendix : the forty tricubes}

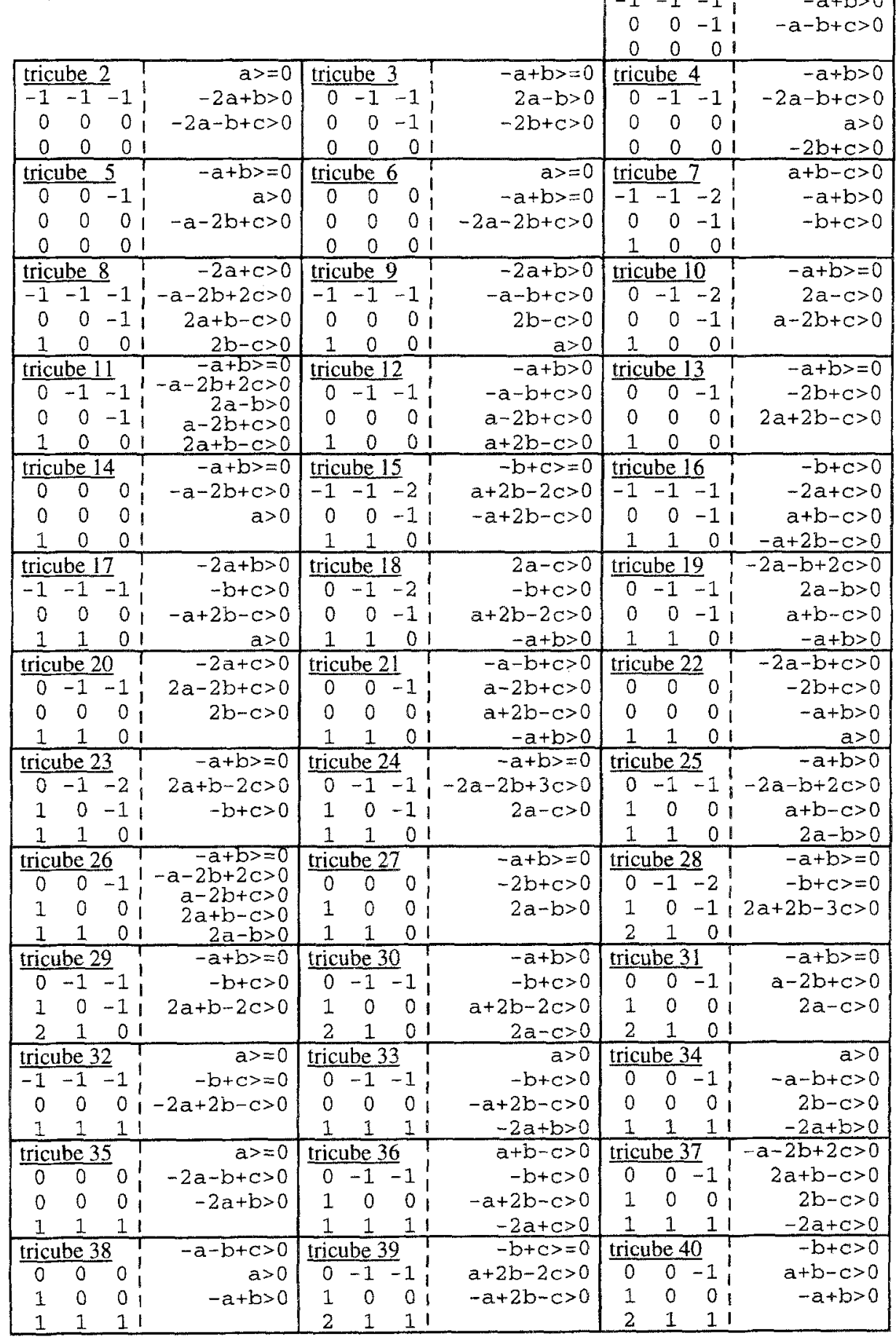

